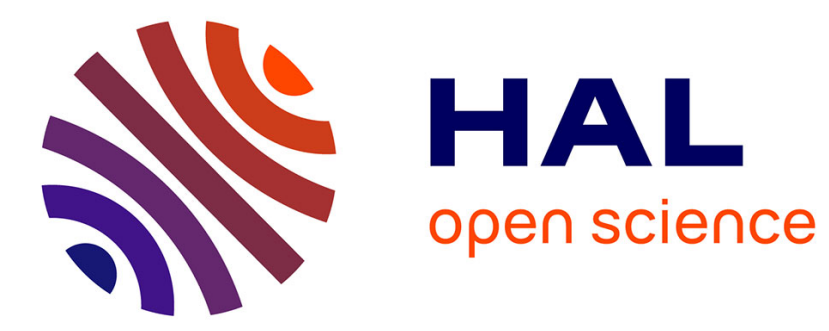

\title{
Opening and retraction of particulate soap films
}

Yousra Timounay, Elise Lorenceau, Florence Rouyer

\section{To cite this version:}

Yousra Timounay, Elise Lorenceau, Florence Rouyer. Opening and retraction of particulate soap films. EPL - Europhysics Letters, 2015, 10.1209/0295-5075/111/26001 . hal-01201687

\section{HAL Id: hal-01201687 \\ https://hal.science/hal-01201687}

Submitted on 28 Sep 2015

HAL is a multi-disciplinary open access archive for the deposit and dissemination of scientific research documents, whether they are published or not. The documents may come from teaching and research institutions in France or abroad, or from public or private research centers.
L'archive ouverte pluridisciplinaire HAL, est destinée au dépôt et à la diffusion de documents scientifiques de niveau recherche, publiés ou non, émanant des établissements d'enseignement et de recherche français ou étrangers, des laboratoires publics ou privés. 


\title{
Opening and retraction of particulate soap films
}

\author{
Yousra Timounay $^{1}$, Elise Lorenceau ${ }^{1}$, Florence Rouyer $^{1}$ \\ 1 Laboratoire Navier, Université Paris-Est, UMR 8205 CNRS, ENPC ParisTech, IFSTTAR, 77454 Champs-Sur- \\ Marne, France
}

\author{
PACS 68.15.+e - liquid films \\ PACS 47.57.Gc - Granular flow, complex fluids \\ PACS $47.55 . \mathrm{nb}$ - Capillary effects, interfacial flows
}

\begin{abstract}
We study for the first time the bursting dynamics of thin liquid films laden with hydrophobic micronic particles either with free or constrained edges. We highlight that the particles can arrange in bilayer or monolayer configurations and explore a range of particles coverage from zero to random close packing. When the particles bridge the two interfaces (monolayer configuration) of free edge films, the hole opens intermittently. For the other cases, we observe constant retraction velocities, modeled by balancing liquid and particles inertia against surface tension as in Taylor-Culick theory. But, this approach is only valid up to a critical value of particles coverage due to the interplay between the interfaces and the friction between particles.
\end{abstract}

Solid hydrophobic particles attached at an air/liquid interface create hybrid interfaces, with a mechanical behavior simultaneously driven by contacts between grains at the 2D granular medium and capillarity $[1,2]$. Previous studies mainly focus on particle rafts [3-6] and coated droplets $[2,7-12]$, where the ratio between the depth of the liquid reservoir and the particles diameter is far larger than 1. However, some recent works on foams stabilized by particles consider particulate films for which the depth of the liquid reservoir is smaller than a particle diameter in an aerated medium $[13,14]$. Yet, particulate films still raise many fundamental questions and practical issues. What is the mechanics of particulate films? Do they deform like liquid films or solid membranes? Do the particles concentration and arrangement at the liquid/air interface affect their mechanics? At the scale of particle laden aqueous foams, a recent work has shown that nanoparticles can stop the coarsening [15]. This behavior has been rationalized using continuum mechanics's framework and deriving a surface elasticity modulus for the particle laden interfaces. Yet, is this analysis also accurate for microparticles or is the framework developed for granular materials more adapted? Moreover, do the particles also modify the other aging processes of foams, namely drainage and film coalescence? These are crucial issues, in particular from a practical point of view, since particle laden foams are encountered in different industries such as cosmetics, food or civil engineering industries. In these applications, aqueous foams are often used as templates to obtain solid aerated materials. The stability of the liquid aqueous foam (fresh material) but also the mechanical and acoustical properties of the solid foam after drying, which depend especially on the fraction of open cells and the apertures sizes of the membranes [16,17], are affected by the presence of the particles. Understanding how the particles affect this aspects is challenging.

In the present work, we study the dynamics of particulate films during their retraction either when they are simply punctured at their center or when the retracting edge is attached to a rigid but mobile boundary. The first mode of retraction, called "free edge" because the retracting edge is the hole boundary, corresponds to the coalescence mechanism at the scale of the foam. The second mode of retraction, called "constrained edge", is similar to film shrinkage during the topological rearrangements $\mathrm{T} 1$ and $\mathrm{T} 2$ at the scale of the foam, due to coarsening or shear.

Thin bare soap films are known to open at constant velocity [18] until total disappearance as predicted by the well-know Taylor-Culick law where a balance of the rim inertia and the capillary forces is assumed. But, in the cases of viscous films [19,20] and elastic surfaces [21], deviations from this law are observed. For particulate films, the high packing of grains at the interface might lead to jamming, thus to a freezing of the retraction dynamics. We thus explore a range of $2 \mathrm{D}$ solid fractions from zero 
to close packing. Different retraction dynamics (intermittent or inertial) are observed depending on the particle configuration and the constraints at the edge.

The liquid used to create the particulate films is an aqueous solution of Sodium Dodecyl Sulfate (SDS) at the critical micelle concentration [22]. This concentration is chosen because any increase of the SDS concentration results in an increase of the film's stability but also leads up to a decrease of the hydrophobicity of the particles in contact with the solution. The CMC therefore happens to be a good compromise between this two aspects. The liquid's volumetric mass density is $\rho_{l}=1000 \mathrm{~kg} / \mathrm{m}^{3}$ and its surface tension is $\gamma=36 \mathrm{mN} / \mathrm{m}$. The particles, in turn, are monosized polystyrene beads (Dynoseeds from Microbeads) with a volumetric mass density $\rho_{P}=1050 \mathrm{~kg} / \mathrm{m}^{3}$. Particles with four different diameters $D_{p}$ are used: 20, 40, 140 and $250 \mu \mathrm{m}$. To modify their wetting properties, the particles have been immersed into a mixture made up of octane solution and dissolved silane. After the silanization, the contact angle $\theta$ between the particles and the $\mathrm{SDS}$ solution at the $\mathrm{CMC}$ is equal to $95^{\circ} \pm 10^{\circ}$ making them slightly hydrophobic.

To create particulate films, we immerse and withdraw a frame made of chrome wires of $60 \mu \mathrm{m}$ diameter from a container filled with the SDS solution with a dense raft of particles on its interface. The surface area of the frame ( $\left.\sim 1 \mathrm{~cm}^{2}\right)$ is in the order of the surface of the liquid films of a dry foam constituted of centimetric bubbles. Moreover, such liquid films are stable during the typical time of one experiment(up to one minute). Using a microscope, we observe two particle configurations on the particulate films. In the monolayer configuration (ML), the particles cross the film and their median plan is within the liquid phase (see FIG 1-a). In the bilayer configuration (BL), the particles are attached to each one of the liquid-air interfaces (see FIG 1-b) and we observe that one interface is generally much more covered in particles than the other one. For one given particle diameter, the particles configuration in the liquid film is perfectly reproducible. We are not able yet to predict this configuration but it seems that it mainly depends on the weight of the particles.

To characterize the particles coverage of the particulate films, we define $\varphi$, the surface fraction of particles per interface, which takes into account the projected surface of the particles on the interfaces:

$$
\varphi=\frac{\alpha \cdot N_{\text {particles }} \cdot S_{\text {particle }}}{2 . S_{\text {film }}}
$$

with $N_{\text {particles }}$ the number of particles present in the film, $S_{\text {particle }}$ the projected surface of a single particle, $S_{\text {film }}$ the surface area of the film and $\alpha=1$ for BL and 2 for ML films, since each particle is attached to both liquid/air interfaces in this configuration.

By exploiting the images prior to the film retraction, we compute the number of particles present in the film (see FIG 1-c). For monolayer films, an image analysis with thresholding of gray levels and circularity as criteria

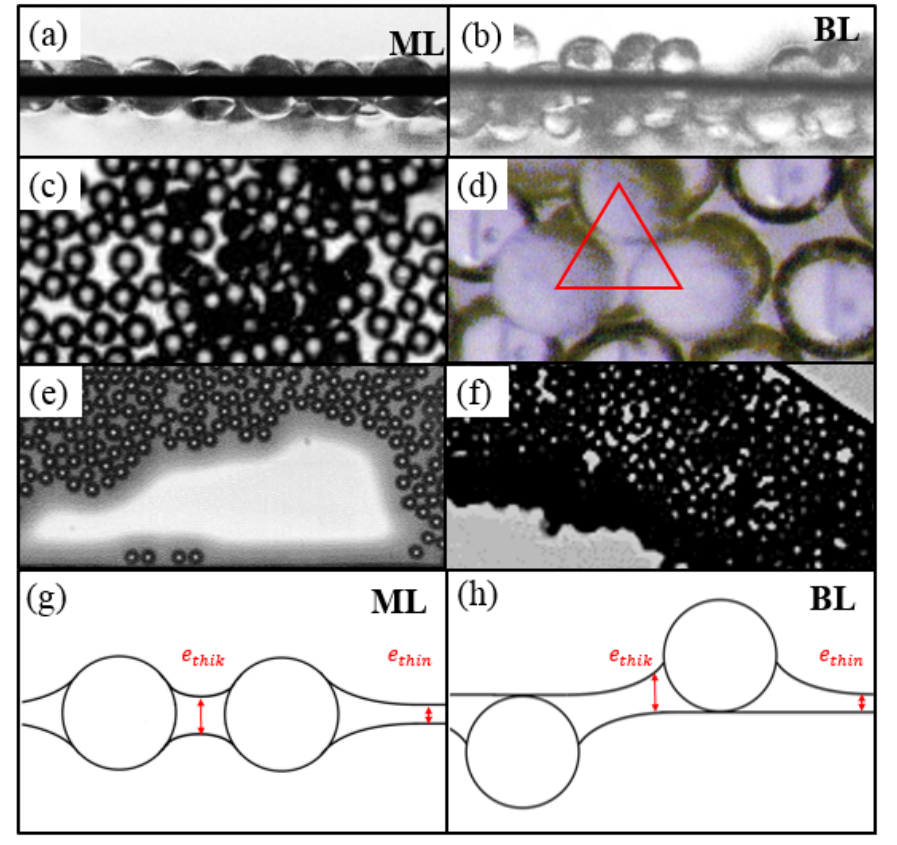

Fig. 1: Pictures of particulate films. (a) Side view of a laden film of $250 \mu \mathrm{m}$ 's particles in the monolayer configuration. (b) Side view of a laden film of $140 \mu \mathrm{m}$ 's particles in the bilayer configuration. The black horizontal line corresponds to the frame wire. (c) Top view of a laden film of $140 \mu \mathrm{m}$ 's particles in the bilayer configuration. (d) A close up on the area where particles of $140 \mu \mathrm{m}$ are attached to both interfaces. (e) A coloured film with $250 \mu \mathrm{m}$ 's particles showing the variation in liquid's thickness. (f) Hexagonal film with $140 \mu \mathrm{m}$ 's particles with a dark rim along the expanding hole. (g) and (h) are schematic side views presenting the film and the trapped particles respectively in the monolayer and the bilayer configuration.

counts the exact number of particles yielding to an incertitude over $\varphi$ less than $2 \%$. For bilayer films, we use the same method as above in the areas where the particles are present only on one single interface. But for the areas where the particles are present on both interfaces, we consider that the random close packing for hard disks $\left(\varphi_{R C P}=0.82 \pm 0.1[23]\right)$ is achieved relying on experimental observations (see FIG 1-d). Adding these two contributions, we obtain the total number of particles with an incertitude over $\varphi$ typically less than $5 \%$.

The thickness of the liquid film $e$ is measured by light interference spectroscopy (Ocean Optics spectrometer used in transmission). The thickness is proportional to the wavelengths for which intensity peaks are observed. A light absorbance method is also used on films colored (see FIG 1-e) with the Acid blue dye (from Fluotechnik). The typical liquid film thickness of bare liquid films, $e_{t h i c k}$ varies between 40 and $50 \mu \mathrm{m}$ for all the frames we used. On particulate films, $e$ is very heterogeneous and ranges from $e_{\text {thick }} \approx 45 \mu \mathrm{m}$ in the vicinity of the particles to $e_{\text {thin }} \approx 5 \mu \mathrm{m}$ where there is no particle (see FIG 1-e, 1-g and 1-h). This heterogeneous film thickness is presumably 
due to the fact that the particles need a liquid's reservoir to fulfill the contact angle condition. Assuming that the area of the thick film is proportional to $\varphi / \varphi_{R C P}$, we approximate an average thickness $\left(e_{\varphi}\right)$ as a function of $\varphi, e_{t h i c k}$ and $e_{\text {thin }}$, that meets the good limit for films covered with closely packed particles:

$$
e_{\varphi}=e_{\text {thick }} \frac{\varphi}{\varphi_{R C P}}+e_{t h i n}\left(1-\frac{\varphi}{\varphi_{R C P}}\right)
$$

To study the hole opening in particulate films, we use a hexagonal frame with a side length equal to $5 \mathrm{~mm}$. The film is gently punctured in its center by a thin needle wrapped in absorbing paper that locally thins the film up to its rupture and thus creates a hole that expands radially (see FIG 2-a). When a heterogeneity in particles coverage is noticed, the hexagonal films are divided into eight parts, where different surface fractions of particles and radial velocities of the hole are measured in each part.

To study the retraction of particulate films when one of the edges is constrained, we use rectangular frames; the length is kept constant equal to $1.5 \mathrm{~cm}$ while the width $a$ can either be equal to $2 \mathrm{~mm}, 5 \mathrm{~mm}$ or $9 \mathrm{~mm}$. Once the rectangular film is formed, we gather all of the particles on the right side of the frame using a stick that can slide on the wires of the frame. This stick is is a hollow cylinder whose length, diameter and mass are respectively $1.6 \mathrm{~cm}$, $6 \mathrm{~mm}$ and $0.01 \mathrm{~g}$ and will constitute the constrained edge of the particulate film. To trigger the particulate film's retraction, we puncture gently the bare film in the vicinity of the stick in order that no liquid is collected on this side of the stick. We follow the retraction's velocity of the film perpendicular to the mobile stick (see FIG 2-b). Only experiments with a quite uniform particles coverage are considered. Indeed, if the particles coverage is heterogeneous, the stick will not remain perpendicular to the frame's wires during its motion and it can not be described by a unique velocity. Each rectangular film is thus characterized by one average surface fraction of particles.

All the experiments are followed with a Photron high speed camera placed above the frame and recording at $1000 \mathrm{fps}$ for rectangular films and $2000 \mathrm{fps}$ for hexagonal ones.

Depending on the particles configuration, the holes in the particulate films open differently as can be observed in FIG 3, where the outlines correspond to the hole boundary taken at a regular time interval for ML and BL films, respectively on the left and right sides. Holes created in the center of ML films expand intermittently and can have an anisotropic shape. On the contrary, holes created in the center of BL films expand uniformly in time and space, they are circular. We describe in the following these two antagonist behaviors and explain them in regards to the particle position at the interfaces.

The anisotropic and intermittent expansion of the hole in ML particulate films, triggered by the successive breakages of capillary bridges between the particles advances (a)

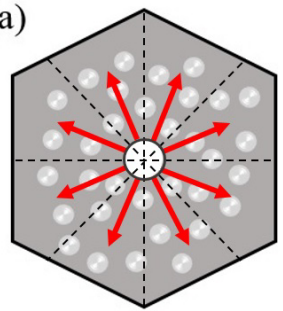

(b)

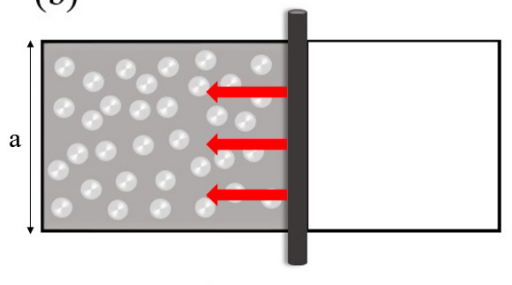

Fig. 2: The two experimental setups. (a) Retraction on an hexagonal frame of a free edge film that has been perforated in the center. The dotted lines delimit the eight slices where the surface fraction of particles is calculated. (b) Retraction on a rectangular frame of a constrained edge film via the motion of the mobile stick triggered by bursting the right side of the film. The red arrows illustrate the rim dynamics (a) and the motion of the stick (b)

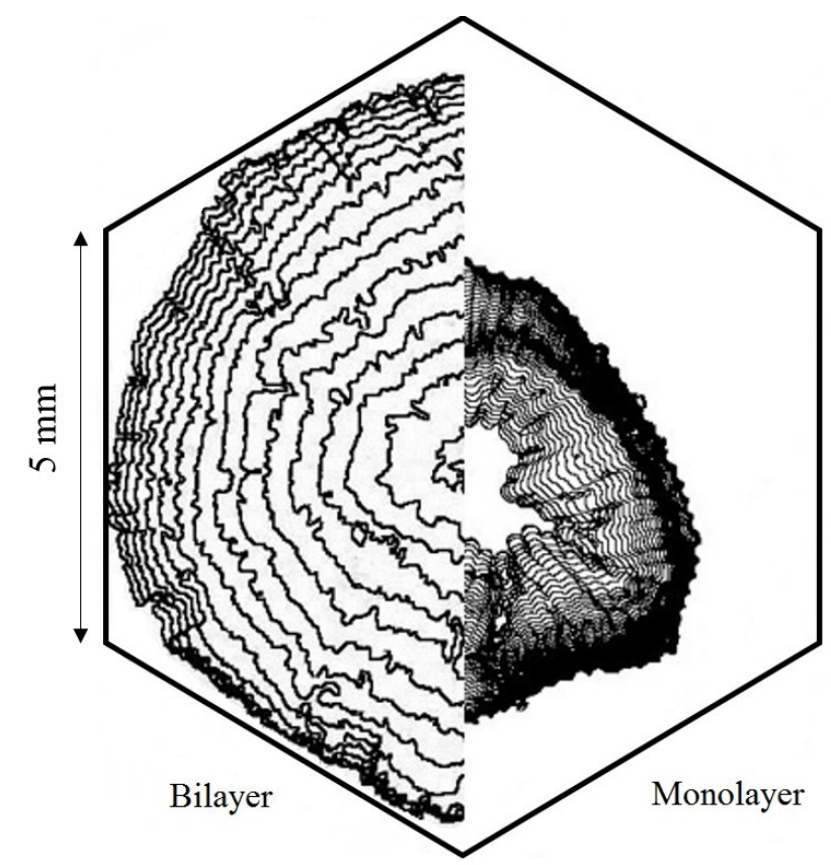

Fig. 3: Superpositions of the rim position in a hexagonal particulate film at every $0.5 \mathrm{~ms}$. On the left, BL configuration with $40 \mu \mathrm{m}$ 's particles at $\varphi=0.55 \pm 0.015$ and on the right, ML configuration with $250 \mu \mathrm{m}$ 's particles at $\varphi=0.66 \pm 0.09$.

with a typical velocity $\approx 0.1 \mathrm{~m} / \mathrm{s}$. This opening pattern is qualitatively similar to the fracture of particle rafts induced by the localized addition of surfactant [24] and to the capillary fingering regime in immersed granular media [25]. Moreover, the hole does not necessarily reach the frame (see the right side of FIG 3). These observations can be explained as follows. In this configuration, the particles bridge the two liquid/air interfaces, which can inhibit the growth of the rim in the vertical direction. Indeed, a rim would not form unless the particles are expelled from the film during the hole opening. To be expelled, a particle needs an energy input greater than its attachment energy to the liquid film $\Delta E_{a} . \Delta E_{a}$ can be approximated by 
comparing the two energy states: the first one being the case where the particle and the liquid film are totally separated and the second one being the case where the median plane of the particle is within the liquid phase. Without taking into account the meniscus around the particle, we estimate $\Delta E_{a}$ to be about $10^{-9} \mathrm{~J}$. Obviously for microparticles, thermal activation is not sufficient to remove particle, but as the hole expands, the particles at the free moving edge acquire a kinetic energy $E_{c}$. For velocity $\sim$ $0.1 \mathrm{~m} / \mathrm{s}: \frac{\Delta E_{a}}{E_{c}} \approx 25$. The expulsion of the particles from the liquid phase is thus not possible which is consistent with our experimental observations. Besides, the capillary bridges between grains can stop the coalescence of ML films: their ruptures would require to open small holes between the particles, which is energetically unfavorable. Indeed, assuming hexagonal close packing of particles, the maximal hole radius is $r=\left(\frac{1}{\sqrt{3}}-\frac{1}{2}\right) D_{p} \approx 20 \mu \mathrm{m}$ which gives a ratio $\frac{r}{e} \approx 0.4$ close to 0.3 , the critical value below which, based on energy considerations, a hole in a soap film would not expand [26]. Moreover, during the opening of the hole, the liquid might be redistributed thus thickening the film and making the ratio $\frac{r}{e}$ even smaller.

On the other hand, for all BL experiments, the film opens uniformly at constant velocity ranging from $0.4 \mathrm{~m} / \mathrm{s}$ to $1.4 \mathrm{~m} / \mathrm{s}$ depending on the surface fraction of particles, as long as the edge of the frame is not reached, as can be seen in the left side of FIG 3. Some particles are ejected from the particulate film during the experiments. It can be explained by comparing the same energies than above. In this case, $\frac{\Delta E_{a}}{E_{c}}$ ranges from 0.3 to 4 depending on the size of the particles. This ratio is much smaller than the one computed in the BL configuration because, in this latter configuration, the particles are attached to only one interface and that their kinetic energy can be significantly larger than in the ML case. However, a darkening of the outer rim of the hole is noticeable, due to the accumulation of particles in this portion of the film (see FIG 1-f). All of these observations coincide qualitatively with what has been observed for bare liquid films despite the presence of solid particles on the interfaces. Indeed, the bursting of a bare liquid film also exhibits a constant velocity that results from the balance between surface tension and inertia of the rim formed at the edge of the hole, $V=\sqrt{\frac{2 \gamma}{e \rho}} \approx$ $1.4 \mathrm{~m} / \mathrm{s}[27-29]$.

From these bursting experiments, we observed that BL films burst in a quite similar way than bare films, and that the velocity of the rim is lower when particles are present in the film. For ML films, the rim can not form because the particles bridge the two interfaces of the film and the only way to increase the hole size is to break the small capillary bridges between the grains, leading to an intermittent opening that can even be stopped.

We now move to the second mode of retraction that is the "constrained edge" geometry where the retracting edge is attached to a mobile stick and thus is forced to be of a constant width. ML and BL films retract in a similar way: the stick velocity reaches a constant value ranging from $0.1 \mathrm{~m} / \mathrm{s}$ to $0.5 \mathrm{~m} / \mathrm{s}$ depending on the surface fraction of particles within few milliseconds. Instead of a rim, folds where liquid and particles are collected appear near the mobile stick. Moreover, the stick can stop before reaching the frame.

More quantitatively, we report $V / V_{0}$ as a function of $\varphi$ on FIG 4, where $V$ is either the hole opening velocity for BL films or the mobile stick velocity and $V_{0}$ the velocity of bare films $(\varphi=0)$ in the same geometry. $V / V_{0}$ decreases with $\varphi$, a feature qualitatively expected from a simple balance of inertia and capillary as in Taylor-Culick model $[28,29]$ since the mass collected per unit time increases with $\varphi$. In the following, we present a model to predict $V$.
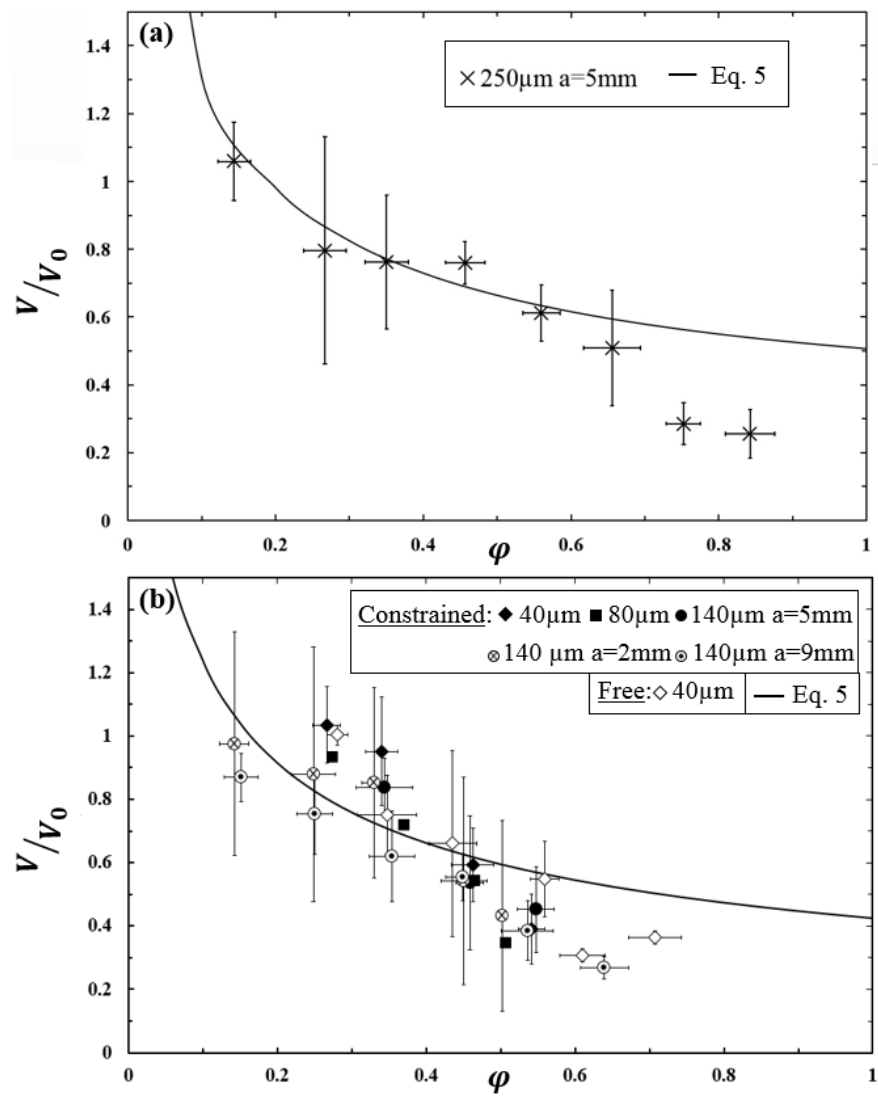

Fig. 4: Retraction velocities normalized by the velocity of bare liquid films as a function of particles surface fraction. Each point of the graph corresponds to an average over an interval of $\varphi$ equal to 0.1 with at least 3 experimental measurements. Data relative to the monolayer configuration are presented in (a) and data relative to the bilayer configuration are presented in (b). Lines are plotted from Equ. 5 with $D_{p}=250 \mu \mathrm{m}$ for (a) and $D_{p}=140 \mu \mathrm{m}$ for (b).

Assuming that both the liquid and the particles are collected at the edge of the retracting film (in the outer rim of the opening hole for BL films or within the folds for constrained edge rectangular films), the variation of the 
mass over time per unit length is :

$$
\frac{d m}{d t}=A \rho_{l} e_{\varphi} V+B \rho_{P} \varphi D_{p} V
$$

where $(A ; B)=\left(1 ; \frac{4}{3}\right)$ for BL films and $(A ; B)=\left(1-\varphi ; \frac{2}{3}\right)$ for ML films. The value of the parameter $B$ in the monolayer configuration is half its value in the bilayer configuration because the particles are attached to the two liquid/air interfaces of the film, so for each interface, we count half the particle mass .

Then, we write the momentum balance per unit of length:

$$
\beta \gamma-F=V \frac{d m}{d t}+m \frac{d V}{d t}
$$

where $\beta$ is a dimensionless coefficient equal to 2 for hexagonal films and smaller than 2 for rectangular films because of the meniscus on the upper air/liquid interface around the stick. As for $F$, it is the global linear resistant force exerted on the rim or on the mobile stick that takes into account the friction of the stick on the wires as well as the friction of the liquid menisci on the wires in the rectangular frame. Determining $F$ is a difficult task as observed in slightly different geometries [30-32], which is far beyond the scope of this paper. Neglecting the contribution of the particles, $F$ in the case of particulate films is assumed to be identical to $F$ in the case of bare liquid films. Moreover, observing that retraction velocities of bare and particulate films are constant over time, it comes:

$$
V=V_{0} \sqrt{\frac{\rho_{l} e_{\text {thick }}}{\rho_{l} e_{\varphi} A+B \rho_{P} \varphi D_{p}}}
$$

For ML constrained edge films, the experimental normalized velocities are in good agreement with Eq.5 as long as $\varphi<0.75$ (see FIG 4-a). This confirms our hypothesis that the particles do not play any role in the resisting force $F$ at low $\varphi$. However, for $\varphi>0.75$, the normalized velocities are lower than expected. At such $\varphi$, approaching $\varphi_{R C P}=0.82$, all particles are in contact with at least three neighbors and the inter-particle friction starts to be significant. Experimental data for BL films covered with small particles, either with constrained or free edges (see FIG 4-b), agree well with Eq.5 for $\varphi<0.5 \pm 0.05$. Because of gravity, the free retracting edge is out of plane for the largest particles, we thus do not represent the experimental points relative to diameter larger than $40 \mu \mathrm{m}$ for the free edge experiments. For $\varphi>0.5 \pm 0.05$ all experimental points are below the theoretical curves. The deviation for BL films can be explained by the inter-particle friction as well. This friction is presumably more intense due to higher number of contacts. Indeed, at given $\varphi$ and particles diameter, the particles are twice as many in the BL configuration than in the ML configuration. This friction can also be more intense due to the absence of lubricated contacts as reported for 2D granular medium at high packing fractions [33]. We emphasize that this second critical value of $\varphi=0.5$ is around the connectivity threshold for particle rafts [34]. Below this threshold, the packing is very loose and particles have few collective effects but once this threshold is reached, the particle assembly may have a granular behavior which results in the deviation from Taylor-Culick law. On another hand, one can argue that this critical $\varphi$ is around half the 2D maximal close packing value, thus the two nearby interfaces interact and considering a projection over the surface area of the film rather than the interfaces to define $\varphi$ might be relevant. Moreover, the deviation from the theoretical curve might be due an increase of the overall viscosity of the films because of the presence of the particles. Indeed, during the opening of viscous films, a transient regime where the rim velocity is smaller than Taylor-Culick velocity is observed [20]. Finally, we remind that we used the surface tension of the solution in Eq.5 rather than an effective surface tension despite the presence of particles on the interfaces. It has been shown that, when adsorbed at an interface, nanoparticles as well as microparticles can change the surface tension of the particle laden interface they are attached to [35-37]. This change becomes significant at high surface coverage $(\sim 0.8)$ and might thus be one of the causes of deviation from Taylor-Culick law that we observe.

\begin{tabular}{|c|l|l|}
\cline { 2 - 3 } \multicolumn{1}{c|}{} & \multicolumn{1}{c|}{ Free edge (FIG 2-a) } & Constrained edge (FIG 2-b) \\
\hline $\begin{array}{c}\text { ML } \\
\text { (FIG 1-a) }\end{array}$ & $\begin{array}{l}\text { Intermittent dynamics } \\
\text { The opening of the hole } \\
\text { can be stopped }\end{array}$ & $\begin{array}{l}\text { Regular dynamics well } \\
\text { described by Taylor } \\
\text { Culick model up to } \\
\varphi=0,75\end{array}$ \\
\hline $\begin{array}{c}\text { BL } \\
\text { (FIG 1-b) }\end{array}$ & $\begin{array}{l}\text { Regular dynamics well described by Taylor Culick model } \\
\text { up to } \varphi=0,5\end{array}$ \\
\hline
\end{tabular}

Fig. 5: Summary of the dynamics of retraction of particulate films depending on the particle position at the interfaces (raw) and the constraints at the edge (column).

To conclude, we have shown that the dynamics of retraction of particulate films depends on the particle position at the interfaces as well as on the constraints on the film edges, the results are summed up in the table on FIG 5. Particles that bridge the two film's interfaces in free edge experiments inhibit the expansion of the hole because the rim formation and the opening of the capillary bridges between the grains are unfavorable. This behavior is quite different from what we have observed for free edge experiments in the bilayer configuration where the opening leads to the total disappearance of the films. In this latter case as well as in the case of constrained edges, the velocities are constant and can be predicted by a modified Taylor-Culick model considering the particles' inertia for low $\varphi$. At large $\varphi$, experimental normalized velocities are lower than expected. Inter-particle friction resists the retraction. The critical $\varphi$ is lower for BL films, suggesting an interaction of the two particulate interfaces through the thin film. The modeling of this granular dissipation 
would require a full characterization of the rheology of the quasi-2D granular medium expected to depend in a non trivial way on both solid fraction and inertia $[38,39]$ which can not be controlled independently in our experiments. To go further, PIV measurements as well as a study on the final state of our experiments are needed.

$$
* * *
$$

The authors would like to thank O. Pitois and X. Chateau for fruitful discussion and C. Méziere for technical help and frames support. We acknowledge CNES for financial support and IFSTTAR for the PhD grant of Y.T.

\section{REFERENCES}

[1] Cicuta P. and Vella D., PHYSICAL REVIEW LETTERS, 102 (2009) .

[2] Lagubeau G., Rescaglio A. and Melo F., PHYSiCAL REVIEW E, 90 (2014)

[3] Horozov T., Aveyard R., Clint J. and Binks B., LANGMUIR, 19 (2003) 2822.

[4] Vella D., Aussillous P. and Mahadevan L., EUROPHYSICS LETTERS, 68 (2004) 212.

[5] Vella D., Kim H., Aussillous P. and Mahadevan L., PHYSICAL REVIEW LETTERS, 96 (2006).

[6] Berhanu M. and Kudrolli A., PHYSiCAL REVIEW LETTERS, 105 (2010)

[7] Aussillous P. and Quere D., NATURE, 411 (2001) 924.

[8] Abkarian M., Protiere S., Aristoff J. M. and Stone H. A., NATURE COMMUNICATIONS, 4 (2013)

[9] Mchale G. and Newton M. I., SOFT MATTER, 7 (2011) 5473

[10] Bormashenko E., CURRENT OPINION IN COLLOID E INTERFACE SCIENCE, 16 (2011) 266.

[11] Planchette C., Lorenceau E. and Biance A.-L., SOFT MATTER, 8 (2012) 2444.

[12] Pitois O., Buisson M. and Chateau X., EUROPEAN PHYSICAL JOURNAL E, 38 (2015) .

[13] Horozov T. S., CURRENT OPINION IN COLLOID \& INTERFACE SCIENCE, 13 (2008) 134.

[14] Rio E., Drenckhan W., Salonen A. and Langevin D., ADVANCES IN COLLOID AND INTERFACE SCIENCE, 205 (2014) 74.

[15] Stocco A., Garcia-Moreno F., Manke I., Banhart J. and LANGEVIN D., SOFT MATTER, 7 (2011) 631.

[16] HoAng M. T. and Perrot C., JOURNAL OF APPLIED PHYSICS, 112 (2012).

[17] Hoang M. T., Bonnet G., Luu H. T. and Perrot C., JOURNAL OF THE ACOUSTICAL SOCIETY OF AMERICA, 135 (2014) 3172.

[18] Frankel S. and Mysels K. J., The Journal of Physical Chemistry, 73 (1969) 3028.

[19] Debregeas G., de Gennes P. and Brochard-Wyart F., SCIENCE, 279 (1998) 1704.

[20] Savva N. and Bush J. W. M., JOURNAL OF FLUID MECHANICS, (2009).
[21] Petit P. C., Le Merre M. and Biance A. L., Journal of Fluid Mechanics, 774 (2015) R3.

[22] Mukerjee P. and Mysels K. J., National Bureau of Standards of NSRDS-NBS 36, Washinglon, DC 20234, (1971)

[23] Berryman J. G., Physical Review A, 27 (1983) 1053.

[24] Vella D., Kim H.-Y., Aussillous P. and Mahadevan L., Physical review letters, 96 (2006) 178301.

[25] Holtzman R., Szulczewski M. L. and Juanes R., Physical review letters, 108 (2012) 264504.

[26] De Vries A., Recueil des travaux chimiques des PaysBas, 77 (1958) 383

[27] De Gennes P.-G., Brochard-Wyart F., Quéré D., Fermigier M. and Clanet C., Gouttes, bulles, perles et ondes (Belin Paris) 2002.

[28] TAYlor G., Proceedings of the Royal Society of London. Series A. Mathematical and Physical Sciences, 253 (1959) 313.

[29] Culick F., Journal of applied physics, 31 (1960) 1128.

[30] Bico J., Ashmore-Chakrabarty J., McKinley G. H. and Stone H. A., PHYSICS OF FLUIDS, 21 (2009) .

[31] Eggers J., Kerswell R. R. and Mullin T., PHYSICAL REVIEW E, 87 (2013).

[32] Cantat I., Physics of Fluids (1994-present), 25 (2013) 031303.

[33] Takehara Y. and Okumura K., Physical review letters, 112 (2014) 148001.

[34] Berhanu M. and Kudrolli A., Physical review letters, 105 (2010) 098002.

[35] Garbin V., Crocker J. C. and Stebe K. J., Journal of colloid and interface science, $\mathbf{3 8 7}$ (2012) 1.

[36] Stocco A., Drenckhan W., Rio E., Langevin D. and Binks B. P., Soft Matter, 5 (2009) 2215.

[37] Lagubeau G., Rescaglio A. and Melo F., Physical Review E, 90 (2014) 030201.

[38] Zhang J., Majmudar T. S., Sperl M. and Behringer R. P., SOFT MATTER, 6 (2010) 2982.

[39] Fall A., Ovarlez G., Hautemayou D., Mzire C., Roux J.-N. and ChevolR F., Journal of Rheology, $\mathbf{5 9}$ (2015) 1065. 